\title{
Evaluation of the clinical value of circulating miR-101, miR-187 and miR-21 in neonatal sepsis diagnosis and prognosis
}

\author{
Rabab F. Salim', Ahmed A. Sobeih ${ }^{2}$ and Heba M. Abd El Kareem ${ }^{1 *}$ (D)
}

\begin{abstract}
Background: Neonatal sepsis is considered as a complicated syndrome, which requires urgent intervention to avoid the unfavorable outcome. Thus, biomarkers that can either distinguish sepsis early or predict sepsis outcome are of critical need. Therefore, the aim of the current study was to investigate the clinical value of miR-187, miR-101, and miR-21 on neonatal sepsis diagnosis and prediction of prognosis. Fifty neonates with sepsis, 30 neonates with SIRS, and 20 healthy neonates were selected. Relative expression levels of the selected miRNAs were quantified by qRT-PCR. Serum CRP and PCT were analyzed.

Results: miR-101 and miR-187 expression levels were elevated in septic neonates compared with SIRS neonates and normal controls. The AUC of miR-101, miR-187, and PCT to predict sepsis diagnosis were $0.908,789$, and 0.856 , respectively. miR-21 expression levels in non-survivors were significantly higher than in survivors. The AUC of miR21, a score of neonatal acute physiology (SNAP-II), and PCT to detect the predictive mortality value were 0.793 , 0.781 , and 0.635 , respectively. Survival analysis revealed that high miR-21 expression levels were related to low survival rates. miR-21 and SNAP II were independent risk factors for sepsis mortality, and the AUC of the two combined variables' predictive probabilities was 0.926 and yielded a specificity of $91.2 \%$ and a sensitivity of $81.3 \%$, which was higher than that of either miR-21 or SNAP II.
\end{abstract}

Conclusion: miR-101 might function as a hopeful diagnostic biomarker for neonatal sepsis. Additionally, miR-21 gained attention to be a valuable predictor for sepsis prognosis especially if combined with SNAP II.

Keywords: Neonatal sepsis, microRNA, Biomarkers, Diagnosis, Prognosis

\section{Background}

Neonatal sepsis is considered as one of the main health problems throughout the world. Neonatal sepsis incidence in developed countries was 1-8/1000 live births, whereas it was approximately three times more in developing countries [1].

Neonatal sepsis can be defined both clinically and/or microbiologically as systemic inflammatory response syndrome (SIRS) caused by infection in the first 4 weeks of life. Initial clinical signs of neonatal sepsis such as tachycardia, fever, and drowsiness are non-specific, and it is easy to misdiagnose with other neonatal diseases as

\footnotetext{
* Correspondence: hebakareem@ymail.com

${ }^{1}$ Biochemistry and Molecular Biology Department, Benha Faculty of Medicine, Benha University, Benha, Egypt

Full list of author information is available at the end of the article
}

metabolic diseases, respiratory distress syndrome, and intracranial hemorrhage [2].

Neonatal infection remains a significant cause of mortality and morbidity, mostly among preterm infants [3]. Various authors reported prematurity, low-birth weight babies, and young age at presentation to have increased mortality in neonatal sepsis [4]. Moreover, maternal factors such as premature rupture of membranes, meconium-stained amniotic fluid, intrapartum fever, foul-smelling amniotic fluid, and instrumental delivery are associated with an increased chance of neonatal sepsis-related mortality [5]. Antepartum hemorrhage and hypertensive diseases during pregnancy are significantly associated with mortality, owing to the increased incidence of preterm neonates and low-birth weight births associated with these disorders. Furthermore, clinical features such as respiratory distress, hypothermia, 
cyanosis, apnea, convulsions, and prolonged capillary refill time have a significant association with mortality in neonatal sepsis, and these may be related to the occurrence of metabolic derangement and cardiovascular collapse [6, 7].

Since the early detection of neonates liable to sepsis may enlarge the therapeutic window and enhance the neonatal outcome, several biomarkers of sepsis have been investigated throughout the last years as possible tools for early identification [8].

Isolation of bacteria from the blood is the gold standard test for neonatal systemic infection; however, the result of culture is not always available before $24-48 \mathrm{~h}$ and is often negative, even in instances of an obvious clinical picture of sepsis [9]. So, biomarkers for distinguishing the sepsis accurately at an earlier stage are urgently needed.

CRP (C-reactive protein) and PCT (procalcitonin) are the most frequently used protein biomarkers for patients with sepsis; however, CRP cannot be used as biomarker for prognosis and early evaluation of patients with sepsis after admission [10] and PCT has low sensitivity and specificity for predicting the mortality of sepsis [11]. Besides, IL-6 is a biomarker for sepsis [12, 13]. However, IL-6 levels are also increased in other non-infectious inflammatory conditions $[14,15]$.

Considering the limitation of the currently established biomarkers, great efforts have been initiated to identify new sepsis biomarkers including circulating microRNAs [16].

MicroRNAs are evolving as new regulators of inflammatory and immune responses [17]. MicroRNAs are a group of endogenous non-coding, single-stranded small RNAs with 18-24 nucleotides (nt) in length. Functionally, microRNAs hinder protein synthesis by directly degrading or inhibiting translation of target genes at the post-transcriptional level [18]. Circulating microRNAs are differentially expressed under different pathological and physiological conditions $[19,20]$. As an expression regulator, microRNAs can target about $60 \%$ of proteincoding genes [21], which exert a vital role in biological processes, such as cell proliferation, apoptosis, differentiation, immunity, and inflammation [22].

MicroRNAs are characterized by their high stability, specificity, and selectivity [23]. Such unique features nominated them to be promising candidate biomarkers, especially for sepsis [24]. In the current study, the relative expression levels of three candidate miRNAs, miR187, miR-101, and miR-21, were analyzed. These miRNAs were selected because of being originated from cells like monocytes, and macrophages, which were activated during microbial infections and, hence, differential expression of these miRNAs [25].

Therefore, the aim of the current study was to investigate the clinical value of miR-187, miR-101, and miR-21 on neonatal sepsis diagnosis and prediction of prognosis.

\section{Methods}

This prospective case-controlled study included 100 neonates, which were categorized into three groups as follows: sepsis group (culture positive group) included 50 neonates with clinical manifestations of sepsis and positive laboratory confirmation of septicemia. SIRS group (culture negative group) included 30 neonates with clinical features suggesting sepsis, but the laboratory investigations were negative. Control group included 20 apparently healthy neonates with neither clinical nor laboratory evidence suggestive of sepsis. These subjects were selected from the neonatal intensive care unit (NICU) of the pediatrics department during the period from May to December 2018. Diagnosis of sepsis was established according to the criteria defined at the 2003 Kunming Neonatal Sepsis Definitions Conference [26]. Preterm neonates, neonates with chromosomal anomalies, intrauterine growth retardation, or perinatal asphyxia, were excluded from the study. The blood samples of the neonates were collected at admission. Serum CRP and PCT values were analyzed. The severity of sepsis was monitored by the score of neonatal acute physiology II (SNAP-II), which is a scoring system includes six items, covering six systems (temperature, $\mathrm{PH}$, oxygenation, blood pressure, the occurrence of multiple seizures, and urine output) [27]. Neonates with sepsis were followed up for 28 days and, hence, were further classified into survivors and non-survivors.

The current study was conducted according to the World Medical Association (WMA) Declaration of Helsinki (2008) and approved by the Research of the Ethical Committee of the Faculty of Medicine. Additionally, written informed consents were obtained from the legal guardians of all neonates registered in the study.

\section{Sample collection}

Anti-cubital venous blood samples $(3 \mathrm{ml})$ were withdrawn from the neonates and were divided into three parts. The first part $(1.5 \mathrm{ml})$ was placed into sterile glass vacutainers for serum separation. The separated sera were used for detection of CRP and PCT. The second part $(1 \mathrm{ml})$ was collected in EDTA tubes for CBC using automated hematology analyzer Sysmex XS-1000i (Sysmex, Japan). The third part $(0.5 \mathrm{ml})$ was collected immediately into RNAprotect Animal Blood Tubes and stored at $-80^{\circ} \mathrm{C}$ for later miRNA extraction.

\section{Extraction of total RNA including microRNAs}

Total RNA including microRNA was extracted from the blood samples $(100 \mu \mathrm{l})$ using RNeasy ${ }^{\circ}$ Protect Animal Blood Kit (Qiagen, Hilden, Germany) in line with the manufacturer's instructions. 


\section{Purity and concentration of RNA}

Eluted RNA was evaluated for purity and concentration using Nanodrop 2000 spectrophotometer (Thermo Fischer Scientific, USA). The readings ratio at $260 \mathrm{~nm}$ and $280 \mathrm{~nm}$ offers an estimate of RNA purity [28]. RNA is considered pure when the A260/A280 ratio is 1.9-2.1.

\section{cDNA synthesis}

Extracted RNA (500 ng) was reverse transcribed by using miScript $^{\bullet}$ II RT Kit (Qiagen, Hilden, Germany) following the manufacturer's instructions. Template RNA was added to every tube containing reverse-transcription master mix, mixed gently, briefly centrifuged, and then kept on ice. Reverse-transcription reaction mixture was then incubated for $60 \mathrm{~min}$ at $37^{\circ} \mathrm{C}$ and for $5 \mathrm{~min}$ at $95^{\circ} \mathrm{C}$ using T100 thermal cycler, Bio-Rad, Singapore. The cDNA samples were diluted in $200 \mu \mathrm{L}$ nuclease-free $\mathrm{H}_{2} \mathrm{O}$ and then stored at -20 for later quantitative realtime PCR (qRT-PCR) analysis.

\section{Relative quantitation of miRNAs}

qRT-PCR was achieved by Rotor-Gene Q (Qiagen, Germany) via miScript SYBR Green PCR kit (Qiagen, Germany). MiRNAs and RNU6-2 (housekeeping gene) miScript Primer Assays were supplied by Qiagen, Germany. Each reaction contained $5 \mu$ l of miScript SYBR Green PCR Master Mix, $1 \mu \mathrm{l}$ of each primer, and $1 \mu \mathrm{l}$ of the cDNA, up to $10 \mu \mathrm{l}$. The optimized thermal profile included an initial denaturation at $95^{\circ} \mathrm{C}$ for $15 \mathrm{~min}, 40 \mathrm{cy}$ cles of denaturation at $95{ }^{\circ} \mathrm{C}$ for $30 \mathrm{~s}$, annealing at $54{ }^{\circ} \mathrm{C}$ for $1 \mathrm{~min}$, and extension at $72^{\circ} \mathrm{C}$ for $30 \mathrm{~s}$. Melting curve analysis was executed to evaluate the specificity of the amplified products. Relative expression of miR-101, miR-187, and miR-21 in each sample were finally detected after normalization with RNU6-2 expression and calculated as $2^{-\Delta \mathrm{Ct}}[29]$. $\Delta \mathrm{Ct}$ was calculated by subtracting the $\mathrm{Ct}$ of the housekeeping gene from that of the target gene. Lower $\Delta \mathrm{Ct}$ values and higher $2^{-\Delta \mathrm{Ct}}$ indicated a higher expression level of the target gene.

\section{Serum CRP and PCT arrays}

CRP was determined by using high sensitivity C-reactive protein (CRP) ELISA (Sigma, Aldrich), and PCT was determined by human procalcitonin ELISA kit (Sigma, Aldrich). Both assays were carried out according to the manufacturer's instructions.

\section{Statistical analysis}

The collected data were tabulated and statistically analyzed using SPSS version 16 software (SPSS Inc., Chicago, ILL Company). Categorical variables were analyzed by chi-square test $\left(\chi^{2}\right)$ or Fisher's exact test (FET). Quantitative data were tested for normality by KolmogorovSmirnov test, supposing normality at $P>0.05$.
Quantitative data were expressed as mean \pm standard deviation (SD), median, and range. Nonparametric data between the two independent groups were compared by Mann-Whitney $U$ test. Differences among three independent means were analyzed by ANOVA for parametric variables or Kruskal-Wallis test (KWT) for nonparametric ones. ROC curves were used to detect the cutoff values of miRNAs with optimum sensitivity and specificity in prediction of diagnosis of sepsis in addition to evaluate their predictive mortality values. To demonstrate whether miR-21 could predict the outcome of the neonates, survival curves were constructed using the KaplanMeier method, and differences in survival were determined by log-rank test. Multivariable logistic regression analysis was done to screen for the independent risk factors for sepsis mortality. Then, the combination of these independent risk factors was performed, and the area under the curve (AUC) for the predictive probabilities of these factors was calculated. The accepted level of significance in this work stated at 0.05. $P<0.05$ was considered significant, and $P$ value $>0.05$ was non-significant.

\section{Results}

Fifty neonates with sepsis, 30 neonates with SIRS, and 20 healthy controls were included in the study. There were no statistically significant differences among the studied groups regarding gender, age, gestational age, weight, and CRP and WBC levels. However, the PCT level was significantly higher in the sepsis group than the SIRS group. Demographic and laboratory characteristics of the different studied groups are shown in Table 1.

\section{Expression levels of miRNAs}

Quantitative RT-PCR revealed that the relative expression levels of miR-101 and miR-187 were significantly increased in the blood of septic neonates compared with SIRS neonates and normal controls. miR-187 was also significantly increased in SIRS neonates compared with normal controls, while miR-101 showed no significant difference between SIRS neonates and normal controls. The expression level of miR-21 was increased in septic and SIRS neonates compared with normal controls, but no significant difference between septic and SIRS neonates was observed (Fig. 1).

\section{Diagnostic value of miRNAs}

ROC curve analyses were generated to compare the diagnostic values of the miRNAs, and the area under curve (AUC) was calculated. Our results revealed that miR-101 had the highest AUC of 0.908 (0.847-0.970), followed by procalcitonin (PCT) with an AUC of 0.856 (0.772-0.941) and miR-187 with an AUC of 0.789 (0.698-0.880). The AUC of CRP was 0.571 (0.442$0.700)$, which was much lower than that of miR-101, 
Table 1 Demographic and laboratory characteristics of the different studied groups

\begin{tabular}{|c|c|c|c|c|}
\hline Variable & Sepsis group $(n=50)$ & SIRS group $(n=30)$ & Control $(n=20)$ & $P$ value \\
\hline \multirow[t]{2}{*}{ Gender male/female } & $25 / 25$ & $14 / 16$ & $11 / 9$ & 0.837 \\
\hline & & Mean \pm SD & & \\
\hline Age (days) & $8.8 \pm 3.37$ & $8.63 \pm 3.35$ & $8.95 \pm 4.501$ & 0.958 \\
\hline Gestational age (weeks) & $37.54 \pm 0.579$ & $37.5 \pm 0.572$ & $37.55 \pm 0.605$ & 0.943 \\
\hline \multirow[t]{2}{*}{ Weight (grams) } & $3.19 \pm 0.34$ & $3.14 \pm 0.29$ & $3.24 \pm 0.39$ & 0.583 \\
\hline & & Median and range & & \\
\hline $\mathrm{CRP}(\mathrm{mg} / \mathrm{L})$ & $10.91(3.21-23.91)$ & $9.955(3.99-21.01)$ & $3.805(2.39-4.81)$ & $0.001^{*}$ \\
\hline PCT $(\mathrm{ng} / \mathrm{ml})$ & $5.53(0.91-13.47)$ & $1.23(0.47-6.23)$ & $0.26(0.10-0.78)$ & $0.001^{*}$ \\
\hline WBC $\left(\times 10^{9} / \mathrm{L}\right)$ & $14.55(2.11-22.6)$ & $14.6(4.21-18.36)$ & $14.65(7.91-18.59)$ & 0.250 \\
\hline
\end{tabular}

${ }^{*} P<0.05$ is significant

PCT, and miR-187. At a cutoff point set at 0.936 , miR101 yielded equal specificity and sensitivity of $84 \%$, while at a cutoff point set at 0.319 , miR-187 yielded a specificity of $76 \%$ and a sensitivity of $72 \%$ (Fig. 2 and Table 2).

miRNA expression levels, CRP, PCT, and SNAP II between sepsis survivors and non-survivors

Based on 28 days follow-up of the sepsis neonates, the current study included 34 neonates which survived 28 days after admission (survivors) and 16 neonates which died within 28 days (non-survivors). miR-21 expression levels and SNAP II in non-survivors were significantly higher than in survivors. In contrast, no significant differences in miR-101 and miR-187 expression levels between sepsis survivors and non-survivors were detected. Similarly, both CRP and PCT did not show significant differences between sepsis survivors and non-survivors (Table 3).

\section{Survival analysis}

Using a cutoff value for miR-21 expression levels of 3.48 relative units, Kaplan-Meier curve revealed that septic neonates with elevated miR-21 expression levels had increased mortality as compared with septic neonates with low miR-21 expression levels $(P<0.05)$. This result indicated that high miR-21 expression levels were related to low survival rates as shown in Fig. 3.

\section{Comparison of the predictive mortality values of miR-21 with SNAP II and PCT}

To investigate the predictive mortality values, ROC curves analysis was generated, and AUC was calculated. Our results indicated that miR-21 had an AUC of 0.793 (0.644-0.942) and at a cutoff point set at 3.48, and miR21 yielded a specificity of $88.2 \%$ and a sensitivity of 62.5\%. AUC of SNAP-II $0.781(0.622-0.940)$ was close to that of miR-21 whereas AUC of PCT was 0.635

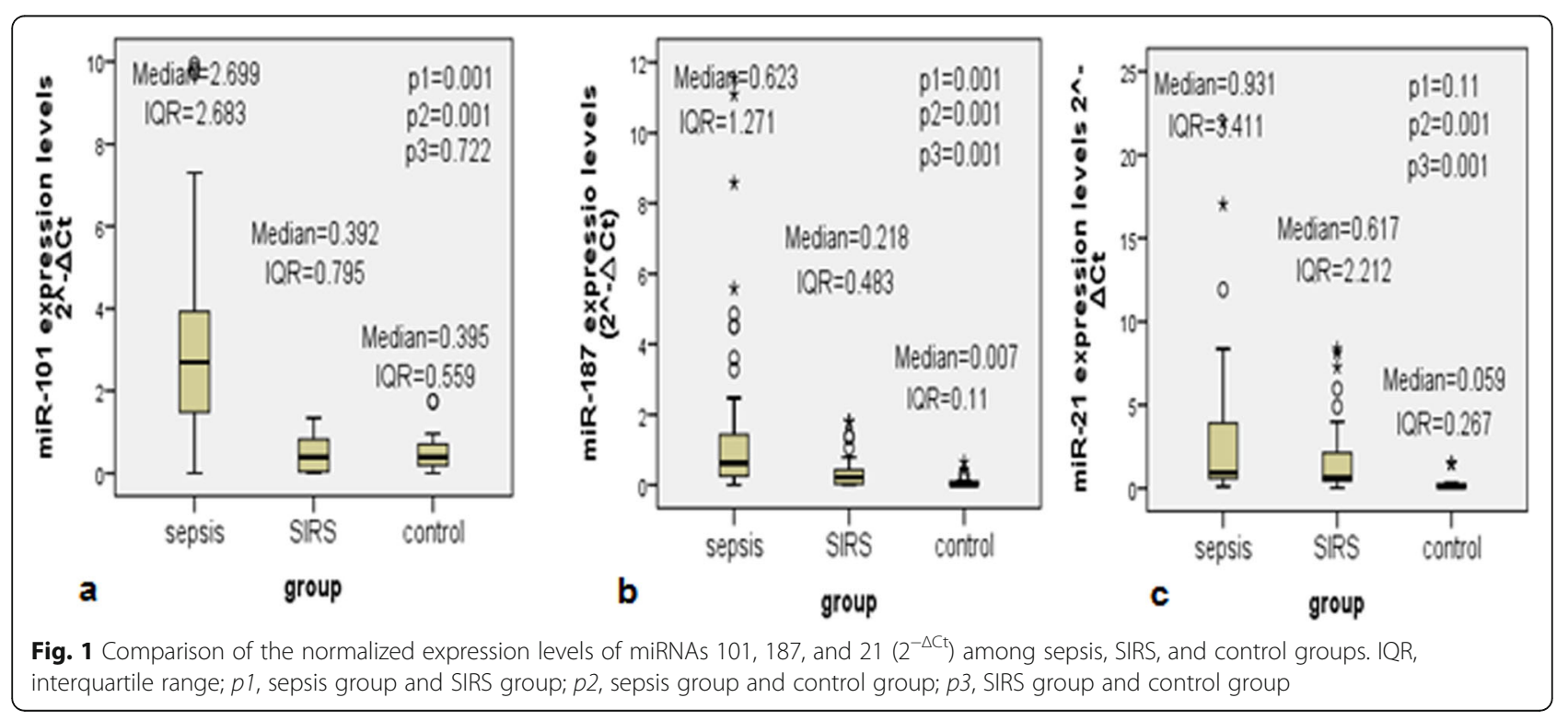



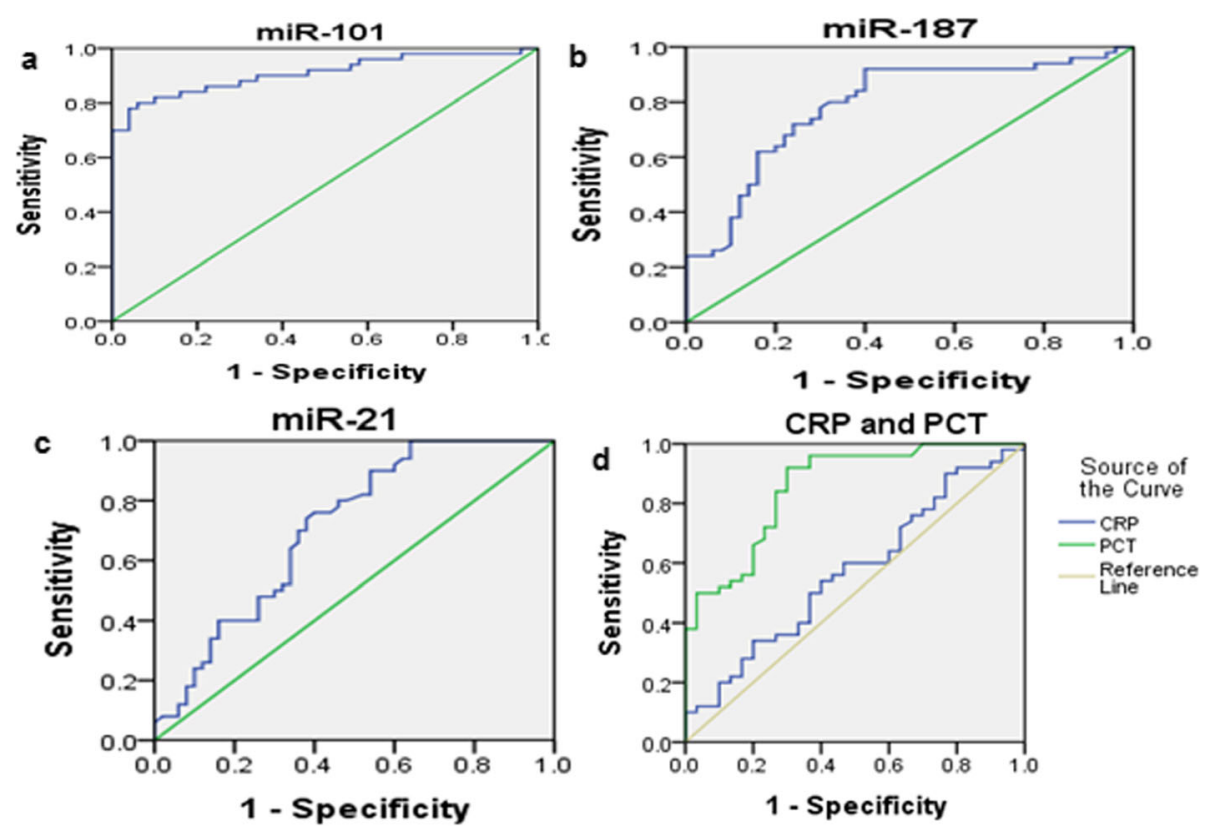

Fig. 2 ROC analyses of miRNAs 101, 187, and 21 normalized expression levels $\left(2^{-\Delta C t}\right)$ and the clinical indicators for discrimination between sepsis and SIRS groups

(0.441-0.829), which was much lower than that of miR21 and SNAP II as shown in Fig. 4.

\section{Multivariable logistic regression analysis}

Binary logistic regression was done to evaluate any possible associations between miR-21 and sepsis outcome. First, univariate logistic regression was done for miR-21 along with age, gestational age, gender, white blood cells (WBCs), CRP, PCT, and score of neonatal acute physiology (SNAP-II), miR-101, and miR-187 (Table 4). This analysis revealed that miR-21, PCT, and SNAP II were significantly different between sepsis survivors and nonsurvivors $(P<0.05)$. These variables were further used in the multivariable logistic regression analysis. Only miR21 and SNAP II have entered the final regression equation. The results showed that miR-21 and SNAP II were independent risk factors for sepsis mortality. The predictive probabilities of miR-21 and SNAP II were calculated from the results of the logistic regression analysis. The ROC curve of the predictive probability of the two variables had an AUC of 0.928 (0.858-0.998), and at a cutoff point of 0.488 , the predictive probability of the combined variables yielded a specificity of $91.2 \%$ and a sensitivity of $81.3 \%$, which was higher than that of miR21and SNAP II. Thus, miR-21and SNAP II combination provided the better predictive capability for sepsis mortality than any of the single variables (Table 5).

\section{Discussion}

The inflammation response of sepsis is initiated by the stimulation of toll-like receptors (TLRs) upon exposure to microbial components such as lipopolysaccharides (LPSs). The activated TLRs trigger a hyperimmune phase with hyper production and release of proinflammatory mediators, causing a cytokine storm and the systemic inflammatory response [24]. Recent evidence suggests that pro- and anti-inflammatory pathways are activated in a parallel manner with the predominance of the proinflammatory response for the first few days of sepsis [30]. If not treated early, the pro-

Table 2 Performance characteristics of miRNAs 101, 187, and 21 expressions $\left(2^{-\Delta C t}\right)$ and clinical indicators in sepsis and SIRS groups

\begin{tabular}{|c|c|c|c|c|c|}
\hline Variables & AUC (95\% Cl) & Cutoff points & Sensitivity (\%) & Specificity (\%) & $P$ value \\
\hline MiR-101 & $0.908(0.847-0.970)$ & 0.936 & 84 & 84 & $<0.001^{*}$ \\
\hline MiR-187 & $0.789(0.698-0.880)$ & 0.319 & 72 & 76 & $<0.001^{*}$ \\
\hline MiR-21 & $0.711(0.609-0.813)$ & 0.732 & 64 & 66 & $<0.001^{*}$ \\
\hline PCT & $0.856(0.772-0.941)$ & 3.520 & 72 & 76.7 & $<0.001^{*}$ \\
\hline CRP & $0.571(0.442-0.700)$ & 10.29 & 56 & 56.7 & 0.29 \\
\hline
\end{tabular}

Cl confidence interval; $* P<0.05$ is significant 
Table 3 Comparison of miRNAs 101, 187, and 21 expression levels $\left(2^{-\Delta C t}\right)$ and the clinical indicators between sepsis survivors and non-survivors

\begin{tabular}{llll}
\hline Variables & $\begin{array}{l}\text { Survivors }(n=34) \\
\text { Median }(\mathrm{IQR})\end{array}$ & $\begin{array}{l}\text { Non-survivors }(n=16) \\
\text { Median }(\mathrm{IQR})\end{array}$ & $P$ value \\
\hline miR-101 & $2.799(3.734)$ & $2.423(1.982)$ & 0.647 \\
MiR-187 & $0.519(1.125)$ & $0.802(2.792)$ & 0.546 \\
miR-21 & $0.756(1.355)$ & $5.067(7.167)$ & $0.001^{*}$ \\
CRP & $10.165(3.76)$ & $14.415(10.48)$ & 0.054 \\
PCT & $4.635(3.54)$ & $8.785(8.55)$ & 0.126 \\
SNAP ॥ & $22(17)$ & $55(36)$ & $0.001^{*}$ \\
\hline
\end{tabular}

${ }^{*} P<0.05$ is significant

inflammatory response abrogates the anti-inflammatory response and, as a result, a hyperimmune or immunosuppression phase progresses where inflammatory mediators overpower the immune system and paralyze it [31] as an adaptive feedback mechanism to control tissue damage during the early hyperinflammatory phase [32]. It is postulated that pro-inflammatory response is responsible for tissue damage and organ failure, while the suppression of anti-inflammatory response increases secondary infections susceptibility [33] and is associated with increased mortality [32].

Early sepsis assessment and diagnosis are critical for timely correction of this complex syndrome [25] especially by using efficient treatment modalities. Circulating miRNAs exist in the blood that could be analyzed rapidly in a clinical setting, in contrast to microbial cultures, which are time-consuming [34]. Such a feature makes it feasible to use miRNAs as beneficial biomarkers for sepsis diagnosis in the clinic [35] and may be valuable in clinical decision-making.
The current study showed that the relative expression levels of miR-101 and miR-187 in sepsis group were significantly higher than the SIRS group and normal controls. In addition, miR-187 was significantly increased in the SIRS group compared with the normal controls, while miR-101 showed no significant difference between the SIRS group and normal controls. A previous study was consistent with our results and reported that miR101 expression was increased in the sepsis patients compared with the healthy neonates [26]. Likewise, our findings were in concordance with Rossato et al. [36] who stated that miR-187 expression, unlike healthy donors, was increased in peripheral blood mononuclear cells (PBMCs) purified from patients with sepsis.

Since sepsis is a SIRS caused by infection, thus, identifying biomarkers that could discriminate true bacterial infection and sepsis from other non-infectious and nonbacterial causes has gained attention as one of the challenges of sepsis diagnosis [24]. In the present study, the ROC curve analysis showed that miR-101 had the highest AUC, followed by PCT and miR-187. The cutoff points of the miRNAs were also analyzed. At a cutoff point set at 0.936 , miR-101 had both high specificity and sensitivity. However, despite being significantly increased in the sepsis group compared with the SIRS group, miR187 yielded lower specificity and sensitivity. Based on these findings, miR-101 exhibited better performance for prediction of neonatal sepsis than that of PCT and miR187. Therefore, our findings suggested that miR-101 might function as a new diagnostic biomarker for neonatal sepsis. This suggestion was attributed to its ability to distinguish sepsis from SIRS more obviously, and its level was just increased in septic neonates but not in SIRS neonates.

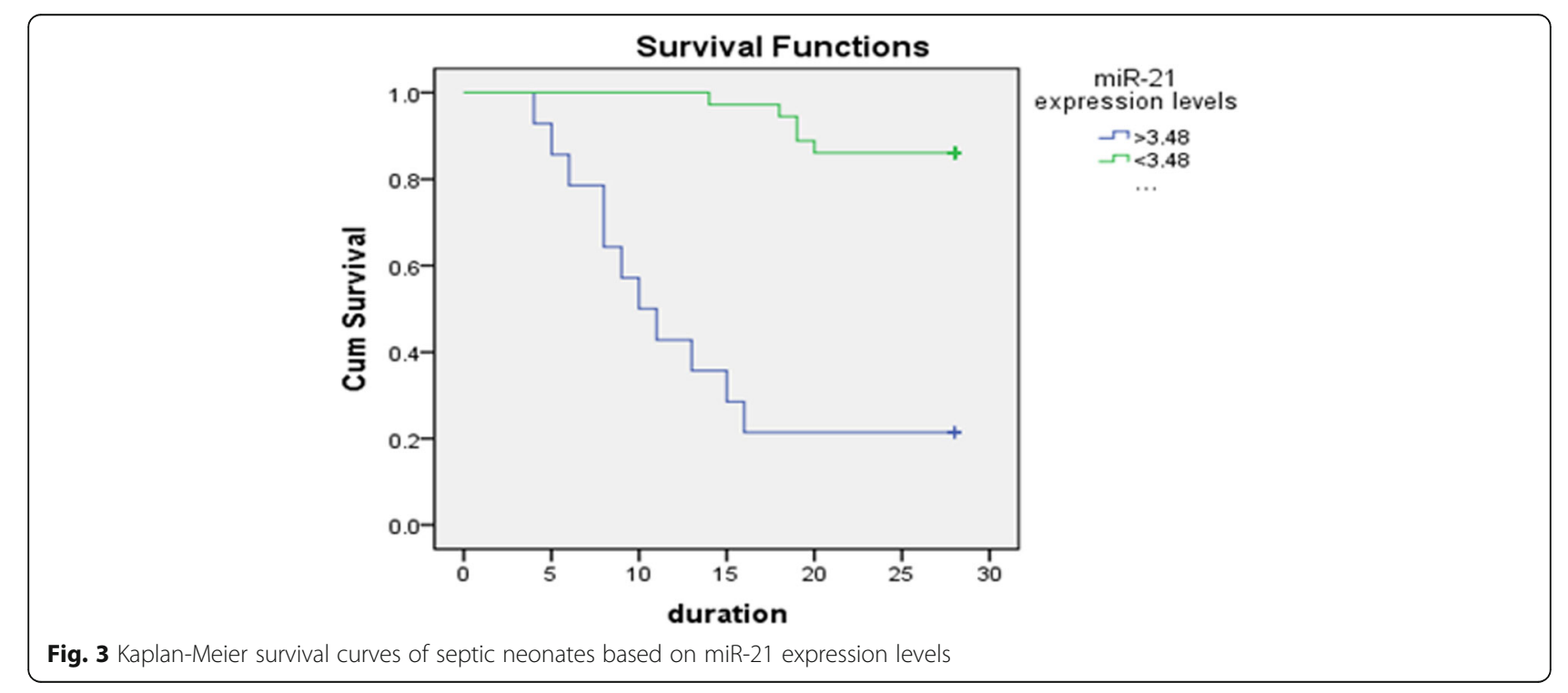



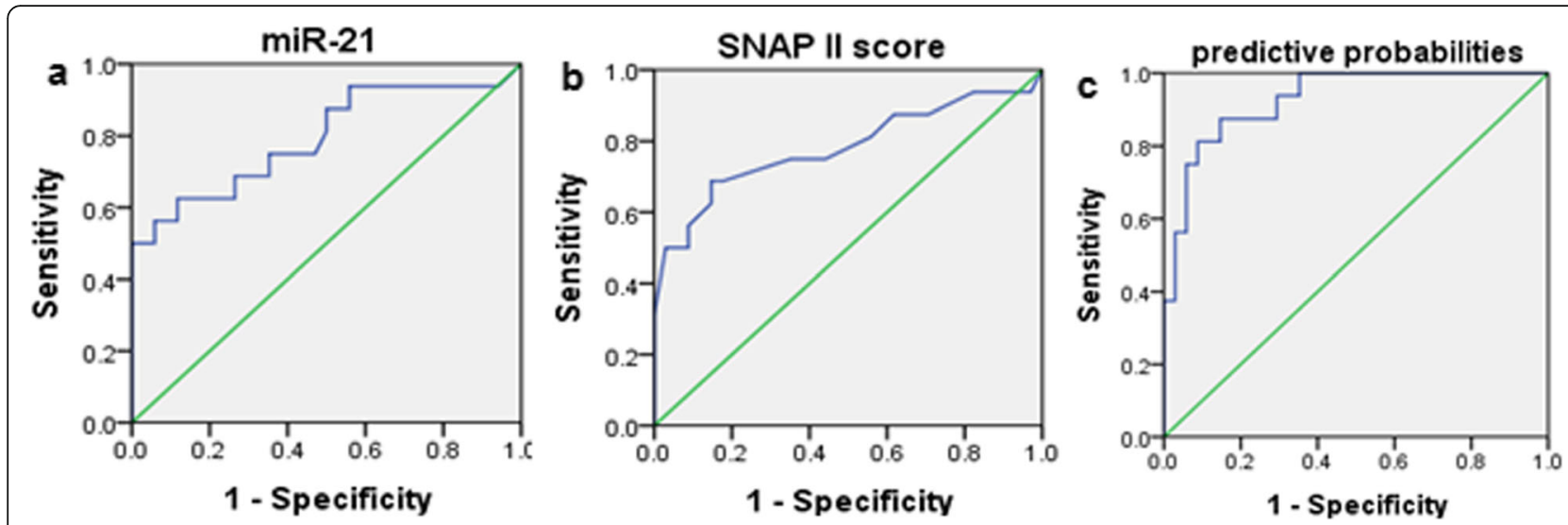

Fig. $4 \mathrm{ROC}$ analyses of miR-21 expression levels $\left(2^{-\Delta C t}\right)$, SNAP II, and predictive probabilities for sepsis survivors and non-survivors. Predictive probabilities calculated from the multivariate logistic regression analysis for the combination of miR-21 levels and SNAP ॥

It is worth mentioning here that miR-101 is induced following cellular activation through multiple TLRs (TLR2, 3, and 4) in response to different microbial stimuli including LPS, polyinosinic-polycytidylic acid, or peptidoglycan, and its induction by LPS is PI3K/Akt pathway dependent [37]. Alternatively, miR-187 is also induced by TLR-mediated cell activation following LPS stimulation but in an IL-10-dependent manner. Rossato et al. [36] proved that IL-10, which is produced systemically during sepsis, synergizes with LPS potentiating miR-187 transcription.

The role of miR-101 and miR-187 during sepsis has been clarified, as well. Zhu et al. [37] identified miR-101 as a critical regulator of the innate immune system through a feedback loop in which TLR-induced miR-101 expression promotes inflammatory response by downregulating $\mathrm{MKP}-1$, which in turn results in prolonged activation of MAPKs that phosphorylate downstream transcription factors and protein kinases and hence subsequent production of proinflammatory cytokines. On the other side, Rossato et al. [32] demonstrated an essential role of miR-187 as a part of a circuit to attenuate the proinflammatory state. It acts as a feedback regulator of LPS response by directly targeting TNF- $\alpha$ mRNA translation and stability and indirectly by decreasing IL-6 and IL-12p40 transcription by silencing the transcription factor $\mathrm{I} k \mathrm{~B} \zeta$.

On the contrary, our findings demonstrated that miR21 expression level in sepsis and SIRS groups was significantly different from that of healthy controls. However, it could not differentiate sepsis from SIRS. Likewise, McClure et al. [32] reported that miR-21 expression was increased in early sepsis and had a sustained an increase in late sepsis. In fact, miR-21 upregulation was mediated via TLR4/MyD88-NF-kB signaling pathway upon LPS challenge [38]. Fujita et al. revealed that the promotor region of pri-miR-21 contains a conserved binding site for AP-1, which can be activated by the TLR4/MyD88

Table 4 Logistic regression analysis of miRNAs 101, 187, and 21 and the clinical parameters between sepsis survivors and nonsurvivors

\begin{tabular}{|c|c|c|c|c|c|c|}
\hline \multirow[t]{2}{*}{ Variables } & \multicolumn{3}{|c|}{ Univariate } & \multicolumn{3}{|c|}{ Multivariate } \\
\hline & $\overline{O R}$ & $\mathrm{Cl}$ & $P$ value & $\overline{O R}$ & $\mathrm{Cl}$ & $P$ value \\
\hline Age & 1.266 & $0.937-1.711$ & 0.125 & & & \\
\hline Gestational age & 8.736 & $0.687-111.147$ & 0.095 & & & \\
\hline Gender & 0.459 & $0.061-3.456$ & 0.450 & & & \\
\hline WBC count & 0.926 & $0.734-1.167$ & 0.514 & & & \\
\hline CRP & 0.884 & $0.688-1.137$ & 0.338 & & & \\
\hline $\mathrm{PCT}$ & 0.491 & $0.253-0.954$ & $0.036^{*}$ & 1.538 & $0.922-2.566$ & 0.100 \\
\hline SNAP I| score & 1.072 & $1.006-1.143$ & $0.032^{*}$ & 0.951 & $0.904-1000$ & $0.049^{*}$ \\
\hline Mir-21 & 4.332 & $1.330-14.105$ & $0.015^{*}$ & 0.422 & $0.200-0.891$ & $0.024^{*}$ \\
\hline MiR-101 & 1.072 & $0.708-1.623$ & 0.742 & & & \\
\hline miR-187 & 1.194 & $0.848-1.681$ & 0.310 & & & \\
\hline
\end{tabular}


Table 5 Performance characteristics of miR-21 expression $\left(2^{-\Delta C t}\right)$, SNAP II, and predictive probabilities in sepsis survivors and nonsurvivors

\begin{tabular}{llllll}
\hline Variables & AUC $(95 \% \mathrm{Cl})$ & Cutoff points & Sensitivity (\%) & Specificity (\%) & $P$ value \\
\hline MiR-21 & $0.793(0.644-0.942)$ & 3.48 & 62.5 & 88.2 & $0.001^{*}$ \\
SNAP II score & $0.781(0.622-0.940)$ & 41 & 68.8 & 85.3 & $0.001^{*}$ \\
PP & $0.928(0.858-0.998)$ & 0.488 & 81.3 & 91.2 & $0.001^{*}$ \\
\hline
\end{tabular}

$P P$ predictive probabilities, $C l$ confidence interval; ${ }^{*} P<0.05$ is significant

pathway. In addition, induction of miR-21 was also dependent on NF-kB, consistent with the presence of a NF-kB binding site in its promotor [39]. Interestingly, miR-21 is overexpressed in many cancers and therefore has been termed as an oncomiR [38].

Prognosis of sepsis is equally important as its diagnosis because it can affect the clinical decision-making pathways [24]. However, it is not possible to deny that the prognosis of sepsis was still unsatisfied. Various studies have exerted efforts to identify biomarkers to predict sepsis outcome, including CRP and PCT. However, the specificities and sensitivities of such biomarkers are not sufficient enough to predict sepsis mortality [40].

Other than miR-101 and miR-187, the current study suggested miR-21 as a potential prognostic biomarker for neonatal sepsis. Our results demonstrated that the relative expression levels of miR-21 in non-survivors were significantly higher than that of survivors. Furthermore, ROC curve analysis of miR-21, to predict the outcome during sepsis, revealed that miR-21 had AUC of 0.793 and yielded $88.2 \%$ specificity and $62.5 \%$ sensitivity when the cutoff point set at 3.48. Moreover, high expression levels of miR21 in the blood of septic neonates were associated with poor outcome and increased mortality rate as represented by Kaplan-Meier survival curves.

Consistent with our results, Wang et al. [41] demonstrated that miR-21 expression levels were significantly higher in the plasma of septic patients with cardiac dysfunction compared with septic patients without cardiac dysfunction and septic cardiac dysfunction was the main cause of death in intensive care units [42].

Noticeable, during the immune response to sepsis, signal transducer and activator of transcription 3 (STAT-3) synergize with CCAAT-enhancer-binding protein beta (C/EBPB) to induce expression of miR-21 and miR$181 \mathrm{~b}$, mediated by IL-6 signals [43]. Induction of both miR-21 and miR-181b in myeloid progenitors increase septic myeloid-derived suppressor cell (MDSC) accumulation by arresting macrophage and dendritic cells differentiation [32]. MDSCs are potently immunosuppressive, suppressing both innate and adaptive immune responses [44-46]. MDSCs suppress the immune response by producing nitric oxide, reactive oxygen species, and proand anti-inflammatory/immunosuppressive cytokines such as TGFB and IL-10 [44, 47, 48]. This sepsis- associated immunosuppression is the major cause of sepsis-related mortality where patients cannot eradicate the invading pathogens and are more susceptible to secondary/opportunistic infections [43].

Unfortunately, the immune response in the neonates with sepsis often favors the immunosuppression state and is biased against the proinflammatory responses probably due to their immature immune response pattern [26].

In line with our results, McClure et al. [32] revealed that in vivo blocking of miR-21 and miR-181b expression reduces MDSC numbers and enhances late sepsis survival. Furthermore, they suggested that this negative path could be attacked to retrieve immunocompetency and improve survival. In contrast, Barnett et al. [49] showed a significant decrease in survival of miR-21 null mice after LPS peritonitis and reported that miR-21 is valuable to survival in mice following LPS-induced peritonitis. However, the conflict observed between these two studies could be attributed to different experimental conditions or the use of different cell types.

As it is known, sepsis is a complex syndrome that involves multiple organs and tissues, and hence a single biomarker is insufficient to reflect either the predictive capability for sepsis prognosis or severity [41]. Therefore, in this study, the predictive probabilities of miR-21 and SNAP II were calculated from the results of the logistic regression analysis. The predictive probability of the combined variables yielded a specificity of $91.2 \%$ and a sensitivity of $81.3 \%$, which was higher than that of either miR-21 or SNAP II. Thus, the combined predictive capability of miR-21 and SNAP II for sepsis mortality was better than any single indicator of them.

Few previous studies to compare our results with, the moderately small sample size, and lack of microorganismrelated analysis were the limitations of this study. Regardless of these limitations, to our knowledge, this study is novel in many aspects. The current study is the first to report the values of miR-101 and miR-187 in distinguishing neonates with sepsis from those with SIRS and, hence, their values as diagnostic biomarkers for neonatal sepsis. Moreover, this study was the first to reveal the value of miR-21 in predicting prognosis of neonatal sepsis based on several findings that were not reported before in any previous studies. Of these findings, not only the relative 
expression levels of miR-21 were significantly higher in sepsis non-survivors than in survivors but also the high expression levels were related to low survival rate. Additionally, miR-21 and SNAP II were independent risk factors for sepsis mortality, and their combined predictive capability for the death of sepsis neonates was better than any single variable of them.

Further prospective studies in a larger scale of the population are essential to confirm our data and to evaluate the dynamic changes of these miRNAs during sepsis. Moreover, the underlying mechanisms and the interaction between these miRNAs and the downstream genes during sepsis are needed to be explored more clearly. This may provide new targets for sepsis treatment.

\section{Conclusion}

To sum up, miR-101 might function as a hopeful diagnostic biomarker for neonatal sepsis with high sensitivity and specificity. On the same pattern, miR-21 gained attention to be a valuable predictor for sepsis prognosis especially if combined with SNAP II.

\begin{abstract}
Abbreviations
Akt: Protein kinase B; AP-1: Activated protein 1; AUC: Area under the curve; C/EBPB: CCAAT-enhancer-binding protein beta; CRP: C-reactive protein; IL6: Interlukin-6; LPSs: Lipopolysaccharides; MAPKs: Mitogen-activated protein kinases; MDSCs: Myeloid-derived suppressor cells; miRNAs: microRNAs; MKP1: Mitogen-activated protein kinase phosphatase 1; MyD88: Myeloid differentiation primary response protein; NF-kB: Nuclear factor kappa beta; PCT: Procalcitonin; PI3K: Phosphatidylinositol-3-kinase; qRT-PCR: Quantitative real time polymerase chain reaction; SIRS: Systemic inflammatory response syndrome; SNAP II: Score of neonatal acute physiology II; STAT-3: Signal transducer and activator of transcription 3; TGFB: Transforming growth factor beta; TLRs : Toll-like receptors; TNF: Tumor necrosis factor
\end{abstract}

\section{Acknowledgements}

Not applicable.

\section{Authors' contributions}

RS and HA carried out the RT-PCR results and participated in the design and drafting of the manuscript, carried out the statistical analysis, and wrote and revised the manuscript. AS diagnosed and properly selected the studied cases. All authors have read and approved the manuscript.

\section{Funding}

This research did not receive any specific grant from funding agencies in the public, commercial, or not-for-profit sectors.

\section{Availability of data and materials \\ The analyzed data are available from the corresponding author on reasonable request.}

\section{Ethics approval and consent to participate}

The study protocol gained approval from the Research of the Ethical Committee of the Faculty of Medicine, in Benha University (reference number is not available because this study approval had been obtained before the ethics committee started numbering). For any details, please contact Professor Dr. Nermeen Adly. Telephone: +201000071033, email: nmadly1@hotmail.com. Written informed consent was obtained from the parent of the participants.

\section{Consent for publication}

Not applicable.

\section{Competing interests}

The authors declare that they have no competing interests.

\section{Author details}

'Biochemistry and Molecular Biology Department, Benha Faculty of Medicine, Benha University, Benha, Egypt. ${ }^{2}$ Pediatrics Department, Benha Faculty of Medicine, Benha University, Benha, Egypt.

Received: 12 October 2019 Accepted: 11 February 2020

Published online: 09 March 2020

\section{References}

1. Thaver D, Zaidi AK (2009) The burden of neonatal infections in developing countries: a review of evidence from community-based studies. Pediatr Infect Dis J. 28(Suppl):S3-S9

2. Radulova P (2010) Neonatal Infections. Diagnostic markers of infection. Akush Ginekol (Sofiia). 49(5):42-51

3. Camacho-Gonzalez A, Spearman PW, Stoll BJ (2013) Neonatal infectious diseases: evaluation of neonatal sepsis. Pediatr Clin N Am. 60:367-389

4. Liang LD, Kotadia N, English L, Kissoon N, Ansermino JM, Kabakyenga J et al (2018) Predictors of mortality in neonates and infants hospitalized with sepsis or serious infections in developing countries: a systematic review. Front Pediatr 6:277

5. Klinger G, Levy I, Sirota L, Boyko V, Lerner-Geva L, Reichman B (2010) Outcome of early-onset sepsis in a national cohort of very low birth weight infants. Pediatrics 125:e736-e740

6. Jajoo M, Kapoor K, Garg LK, Manchanda V, Mittal SK (2015) To study the incidence and risk factors of early onset sepsis in an out born neonatal intensive care unit of India. J Clin Neonatol 4:91-95

7. Bharad RV, Singh CS, Singh LR (2017) Risk factors and immediate outcome of early onset neonatal sepsis. J Med Sci Res 5:21050-21056

8. Ng P (2004) Diagnostic markers of infection in neonates. Arch Dis Child Fetal Neonatal Ed. 89(3):F229-F235

9. Bhandari V, Wang C, Rinder C, Rinder H (2008) Hematologic profile of sepsis in neonates: neutrophil CD64 as a diagnostic marker. Paediatrics. 121(1):129-134

10. Silvester J, Povova P, Coelho L, Almedia E, Moreira P, Fernandes A, Mealha R, Sabino H (2009) Is C-reactive protein a good prognostic marker in septic patients? Intensive Care Med. 35:909-913

11. Pettila V, Hynninen M, Takkunen O, Kuusela P, Vatonen M (2002) Predictive value of procalcitonion and interleukin 6 in critically ill patients with suspected sepsis. Intensive Care Med. 28:1220-1225

12. Harbarth S, Holeckova K, Froidevaux C, Pittet D, Ricou B, Grau GE, Vadas L, Pugin J (2001) Diagnostic value of procalcitonin, interleukin-6, and interleukin-8 in critically ill patients admitted with suspected sepsis. Am J Respir Crit Care Med. 164:396-402

13. Selberg O, Hecker H, Martin M, Klos A, Bautsch W, Kohl J (2000) Discrimination of sepsis and systemic inflammatory response syndrome by determination of circulating plasma concentrations of procalcitonin, protein complement 3a, and interleukin-6. Crit Care Med. 28:2793-2798

14. Jawa RS, Anillo S, Huntoon K, Baumann H, Kulaylat M (2011) Analytic review: interleukin-6 in surgery, trauma, and critical care: part I: basic science. J Intensive Care Med. 26:3-12

15. Jawa RS, Anillo S, Huntoon K, Baumann H, Kulaylat M (2011) Interleukin-6 in surgery, trauma, and critical care part II: clinical implications. J Intensive Care Med. 26:73-87

16. Benz F, Roy S, Trautwein C, Roderburg C, Luedde T (2016) Circulating microRNAs as biomarkers for sepsis. Int J Mol Sci 9(1):17

17. O'Connell RM, Rao DS, Chaudhuri AA, Baltimore D (2010) Physiological and pathological roles for microRNAs in the immune system. Nat Rev Immunol 10:111-122

18. Chen X, Ba Y, Ma L, Cai X, Yin Y, Wang K, Guo J, Zhang Y, Chen J, Guo X, Li Q, Li X, Wang W, Zhang Y, Wang J, Jiang X, Xiang Y, Xu C, Zheng P, Zhang J, Li R, Zhang H, Shang X, Gong T, Ning G, Wang J, Zen K, Zhang J, Zhang CY (2008) Characterization of microRNAs in serum: a novel class of biomarkers for diagnosis of cancer and other diseases. Cell Res. 18:997-1006

19. Shifeng H, Danni W, Pu C, Ping Y, Ju C (2013) Lipi ng Z. Circulating liverspecific miR-122 as a novel potential biomarker for diagnosis of cholestatic liver injury. PLoS One. 8:e73133

20. Abue M, Yokoyama M, Shibuya R, Tamai K, Yamagu-Chi K, Sato I, Tanaka N, Hamada S, Shimosegawa T, Sugamura K, Satoh K (2015) Circulating miR- 
483-3p and miR-21 is highly expressed in plasma of pancreatic cancer. Int J Oncol 46:539-547

21. Zheng N, Yang P, Wang Z, Zhou Q (2017) OncomicroRNAs- mediated tumorigenesis: implication in cancer diagnosis and targeted therapy. Curr Cancer Drug Targets. 17:40-47

22. Bartel DP (2009) MicroRNAs: target recognition and regulatory functions. Cell. 136:215-233

23. Perfetti A, Greco S, Bugiardini E et al (2014) Plasma microRNAs as biomarkers for myotonic dystrophy type 1. Neuromusc Dis 24(6):509-515

24. Inal Ç, Tanrı̈̈ver MD, Erden DD (2016) Novel transcriptional biomarkers for diagnosis and prognosis of sepsis. Acta Medica. 47(1):11-18

25. Wang JF, Yu ML, Yu G, Bian JJ, Deng XM, Wan XJ, Zhu KM (2010) Serum miR-146a and miR-223 as potential new biomarkers for sepsis. Biochem Biophys Res Commun 394(1):184-188

26. Chen J, Jiang S, Cao Y, Yang Y (2014) Altered miRNAs expression profiles and modulation of immune response genes and proteins during neonatal sepsis. J Clin Immunol. 34(3):340-348

27. Chiesa C, Panero A, Osborn JF, Simonetti AF, Pacifico L (2004) Diagnosis of neonatal sepsis: a clinical and laboratory challenge. Clin Chem. 50(2):279-287

28. Wilfinger WW, Mackey K, Chomczynski P (1997) Effect of pH and ionic strength on the spectrophotometric assessment of nucleic acid purity. BioTechniques. 22(3):474-476 478-81

29. Livak KJ, Schmittgen TD (2001) Analysis of relative gene expression data using real-time quantitative PCR and the $2-\Delta \Delta C T$ method. Methods. 25 : 402-408

30. Laszlo I, Trasy D, Molnar Z, Fazakas J (2015) Sepsis from pathophysiology to individualized patient care. J Immunol Res. 510436

31. Sagy M, Al-Qaqaa Y, Kim P (2013) Definitions and pathophysiology of sepsis. Curr Probl Pediatr Adolesc Health Care. 43:260-263

32. McClure C, Brudecki L, Ferguson DA, Yao ZQ, Moorman JP, McCall CE, El Gazzar M (2014) MicroRNA 21 (miR-21) and miR-181b couple with NFI-A to generate myeloid-derived suppressor cells and promote immunosuppression in late sepsis. Infect Immun. 82(9):3816-3825

33. Angus DC, van der Poll T (2013) Severe sepsis and septic shock. N Engl J Med. 369:840-851

34. Essandoh K, Fan GC (1842) Role of extracellular and intracellular microRNAs in sepsis. Biochim Biophys Acta. 2014:2155-2162

35. Zhang TN, Li D, Xia J, Wu QJ, Wen R, Yang N, Liu CF (2017) Non-coding RNA: a potential biomarker and therapeutic target for sepsis. Oncotarget 8(53):91765-91778

36. Rossato M, Curtale G, Tamassia N, Castellucci M, Mori L, Gasperini S, Mariotti B, De Luca M, Mirolo M, Cassatella MA, Locati M, Bazzoni F (2012) IL-10induced microRNA-187 negatively regulates TNF- , IL-6, and IL-12p40 production in TLR4-stimulated monocytes. Proc Natl Acad Sci U S A 109(45): E3101-E3110

37. Zhu QY, Liu Q, Chen JX, Lan K, Ge BX (2010) MicroRNA-101 targets MAPK phosphatase-1 to regulate the activation of MAPKs in macrophages. J Immunol 185(12):7435-7442

38. Sheedy FJ, Palsson-McDermott E, Hennessy EJ, Martin C, O'Leary JJ, Ruan Q, Johnson DS, Chen Y, O'Neill LA (2010) Negative regulation of TLR4 via targeting of the proinflammatory tumor suppressor PDCD4 by the microRNA miR-21. Nat Immunol. 11(2):141-147

39. Fujita S, Ito T, Mizutani T, Minoguchi S, Yamamichi N, Sakurai K, Iba H (2008) miR-21 Gene expression triggered by AP-1 is sustained through a doublenegative feedback mechanism. J Mol Biol 378:492-504

40. Wang $H$, Zhang P, Chen W, Feng D, Jia Y, Xie $L$ (2012) Serum microRNA signatures identified by Solexa sequencing predict sepsis patients' mortality: a prospective observational study. PLoS One. 7(6):e38885

41. Wang $H$, Bei $Y$, Shen S, Huang P, Shi J, Zhang J, Sun Q, Chen Y, Yang Y, Xu T, Kong X, Xiao J (2016) miR-21-3p controls sepsis-associated cardiac dysfunction via regulating SORBS2. J Mol Cell Cardiol 94:43-53

42. Romero-Bermejo FJ, Ruiz-Bailen M, Gil-Cebrian J, Huertos-Ranchal MJ (2011) Sepsis-induced cardiomyopathy. Curr Cardiol Rev. 7(3):163-183

43. McClure C, McPeak MB, Youssef D, Yao ZQ, McCall CE, El Gazzar M (2017) Stat3 and C/EBP $\beta$ synergize to induce miR-21 and miR-181b expression during sepsis. Immunol Cell Biol. 95(1):42-55

44. Gabrilovich DI, Nagaraj S (2009) Myeloid-derived suppressor cells as regulators of the immune system. Nat Rev Immunol. 9:162-174

45. Brudecki L, Ferguson DA, McCall CE, El Gazzar M (2012) Myeloid-derived suppressor cells evolve during sepsis and can enhance or attenuate the systemic inflammatory response. Infect Immun. 80:2026-2034
46. Kong YY, Fuchsberger M, Xiang SD, Apostolopoulos V, Plebanski M (2013) Myeloid derived suppressor cells and their role in diseases. Curr Med Chem. 20:1437-1444

47. Youn Jl, Nagaraj S, Collazo M, Gabrilovich DI (2008) Subsets of myeloidderived suppressor cells in tumor-bearing mice. J Immunol. 181:5791-5802

48. Delano MJ, Scumpia PO, Weinstein JS, Coco D, Nagaraj S, Kelly-Scumpia KM et al (2007) MyD88-dependent expansion of an immature GR-1(+)CD11b(+) population induces T cell suppression and Th2 polarization in sepsis. J Exp Med. 204:1463-1474

49. Barnett RE, Conklin DJ, Ryan L, Keskey RC, Ramjee V, Sepulveda EA, Srivastava S, Bhatnagar A, Cheadle WG (2016) Anti-inflammatory effects of miR-21 in the macrophage response to peritonitis. J Leukoc Biol. 99(2):361371

\section{Publisher's Note}

Springer Nature remains neutral with regard to jurisdictional claims in published maps and institutional affiliations.

\section{Submit your manuscript to a SpringerOpen ${ }^{\circ}$ journal and benefit from:}

- Convenient online submission

- Rigorous peer review

- Open access: articles freely available online

- High visibility within the field

- Retaining the copyright to your article

Submit your next manuscript at $>$ springeropen.com 